\title{
Obesogens and Obesogenic Environment: New Targets for Prevention of Global Obesity
}

\author{
Yun Feng ${ }^{1, a^{*}}$ and Zhutao Wang ${ }^{1, b}$ \\ ${ }^{1}$ Department of Pharmacology, School of Medicine, Jiangsu University, Zhenjiang, Jiangsu \\ 212013, China \\ afengyun76@126.com, bwangzhutao95@qq.com
}

Keywords: Obesity; Obesogens; Obesogenic environment

\begin{abstract}
Obesity refers to abnormal biological status characterized by overweight or having excess adipose tissue, defined as BMI (body mass index) over 30 (or 28 for Chinese). With increasing population of overweight and obesity, it has been a great threat to human health, in that it contributed to illnesses such as endocrine diseases, metabolic diseases and so on. Researches on obesity mainly focus on lifestyle and diet changes, blaming obesity mainly on sedentary lifestyle and high calorie uptake, which could not lead to infants and domestic animals getting fat completely and actions to prevent obesity based merely on such theory have not made great progress. Considering specific chemicals have negative effect on differentiation, proliferation and metabolism, recent researches pay more attention to obesogenic environments and obesogens and their mechanism of action. Such researches provide new targets for preventing global obesity, making it closer to eliminating such threat.
\end{abstract}

\section{Introduction}

With the population of overweight and obesity around the world increasing from 857,000,000 in 1980 to 2,100,000,000 in 2013, obesity has been a worldwide public health concern, in that it poses a great threat to human health, especially facilitating cardiovascular, endocrine and metabolic diseases. Sedentary lifestyle and high calorie uptake resulting in an increase in overweight and obesity rates do not contribute to the obesity of infants, children and animals in close contact with humans. Actions to prevent obesity based on blaming it on sedentary lifestyle and high calorie uptake have not made it completely so far. Against such background, it is urgent to figure out more potential factors for obesity. Considering that obesity is characterized by the ascent of weight and body fat, researchers have taken into account whether some environmental factors may affect the energy and material metabolism in the body (lipid metabolism, glycometabolism, and protein metabolism), which could lead to this phenomenon.

\section{Obesogens and Obesogenic Environment}

Obesogens. Obesogens refers to all chemicals that disrupt normal development and balance of adipose tissue metabolism, including diethylstilbestrol (DES), bisphenol A (BPA), organotin (e.g:tributyltin (TBT)), nonylphenol (NP), phthalates (PAEs), chlorpromazine (Cp), persistent organic pollutants (POPs), Cd2+, ceramide and tolylfluanid (TF) and so on, which in some cases, may lead to obesity. These chemicals could affect weight and forming of adipose tissue. According to the mechanism, obesogens could be divided into two kinds: direct obesogens and indirect obesogens. Direct obesogens could disrupt signal pathways and then lead to follow-up effects, like disrupting differentiation of adipocyte, affecting glycometabolism, enhancing the lipid accumulation, disrupting other hormones or increasing appetite, etc. Indirect obesogens could contribute to obesity via some intermediary agent.

The possibility that obesity may result in part due to chemicals was proposed in 2002, when Baillie-Hamilton found that specific chemicals play a great role in the obesity forming process. Over the last 40 years, consumption of industrial chemicals and the rate of obesity have risen at a similar speed. In 2006, Bruce Blumberg suggested that these chemicals be collectively named obesogen based 
on the similar effects of these chemicals [1]. The introduction of this technical term comprehensively summarizes all environmental chemicals that adversely affect the function of adipose tissue. From the perspective of lipometabolism, obesogen alters fat reserves, and lipid homeostasis, thereby interfering with energy balance and the regulation of appetite and satiety, and thus increasing the accumulation of fat and eventually causing obesity. Scholars who support the concept of obesogen point out some chemicals, such as pesticides, food additives and plasticizer, that can cause a living organism to store more fat inside than usual, in other words, cause overweight or obesity. Domestic scholars suggested the word obesogen as environmental fattening agent in Chinese[2-12].

Table 1: Obesogens and their mechanisms

\begin{tabular}{|c|c|c|c|}
\hline Obesogens & \multicolumn{2}{|c|}{ Chemicals } & Mechanism \\
\hline \multirow{19}{*}{$\begin{array}{c}\text { Direct } \\
\text { obesogens }\end{array}$} & \multirow{2}{*}{\multicolumn{2}{|c|}{ DES }} & Disrupting differentiation of fibroblasts into adipocytes. \\
\hline & & & Enhancing glucose uptake. \\
\hline & \multicolumn{2}{|l|}{ BPA } & Releasing less glucagon. \\
\hline & & & Affecting adipocyte proliferation and differentiation. \\
\hline & \multirow{2}{*}{\multicolumn{2}{|c|}{ TBT }} & Altering DNA methylation. \\
\hline & & & Promoting adipogenesis \\
\hline & \multicolumn{2}{|l|}{ NP } & Disrupting differentiation of fibroblasts into adipocytes. \\
\hline & \multicolumn{2}{|l|}{ PAEs } & Affecting glycometabolism. \\
\hline & \multicolumn{2}{|l|}{$\mathrm{Cp}$} & Increasing appetite. \\
\hline & & OCPs & Antiandeogenic. \\
\hline & \multirow{5}{*}{ POPs } & PCB & Disrupting differentiation of fibroblasts into adipocytes. \\
\hline & & PBDEs & Disrupting differentiation of fibroblasts into adipocytes. \\
\hline & & $\mathrm{DFC}$ & Disrupting glucose and lipid metabolism. \\
\hline & & PFCS & Change expression of genes. \\
\hline & & PAH & Decreasing methylation of PPAY $\gamma$, and increase its expression \\
\hline & \multicolumn{2}{|l|}{$\mathrm{Cd}$} & Accelerating glycometabolism. \\
\hline & \multicolumn{2}{|l|}{ Ceramide } & Resulting in insulin resistance. \\
\hline & \multicolumn{2}{|l|}{$\mathrm{TF}$} & Resulting in insulin resistance. \\
\hline & \multicolumn{2}{|l|}{$\mathrm{CO} 2$} & Increasing mass of WAT(white adipose tissue) \\
\hline \multirow{2}{*}{$\begin{array}{c}\text { Indirect } \\
\text { obesogens }\end{array}$} & \multicolumn{2}{|c|}{ PM10/PM2.5 } & $\begin{array}{l}\text { Reducing sunlight reaching creatures on earth would contribute } \\
\text { to vitamin D deficiency, which would inhibit the differentiation of } \\
\text { pre-adipocytes. }\end{array}$ \\
\hline & \multicolumn{2}{|c|}{$\mathrm{Cd}, \mathrm{Ca}$} & $\begin{array}{l}\text { Disrupting gut microbial ecology, which would alter microbially } \\
\text { mediated effects on ADME(absorption, distribution,metabolism } \\
\text { and excretion). }\end{array}$ \\
\hline
\end{tabular}

Direct obesogens could disrupt signal pathways, mimicking hormones, activating or inhibiting some special receptors, which would alter biological processes and cause obesity. Mediation is need when indirect obesogens contribute to obesity. Most obesogens are basic or common raw material in industry. 


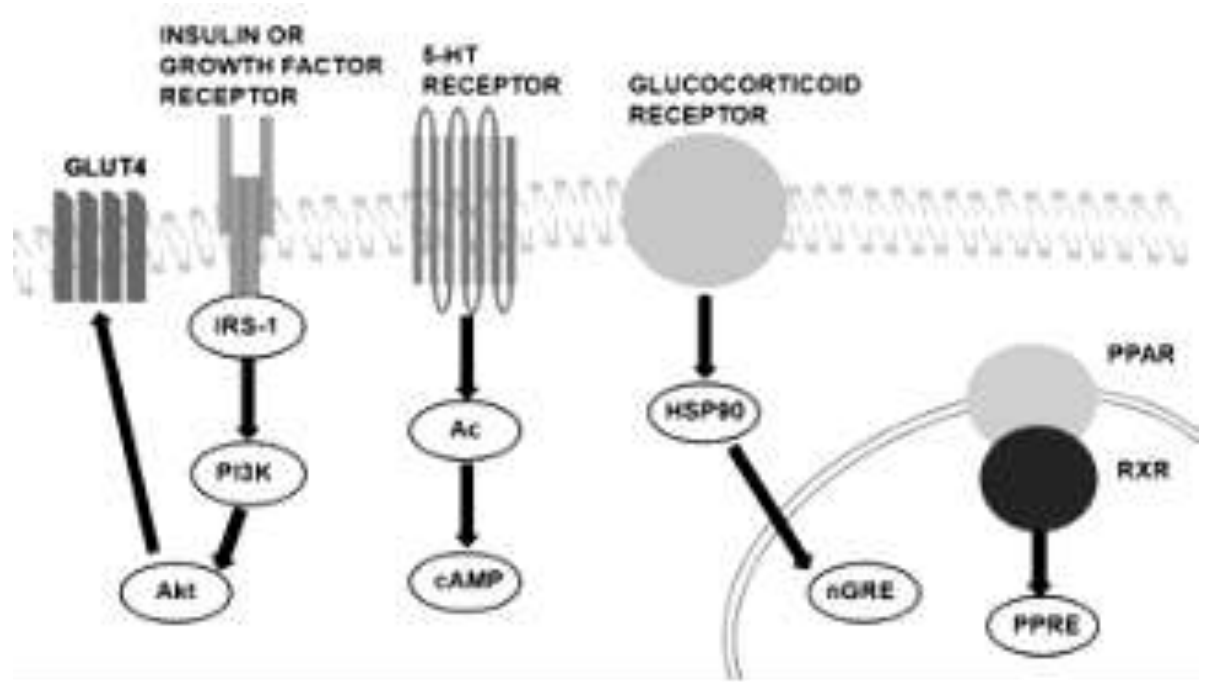

Figure 1. Typical direct obesogens disrupted obesity relating signal pathways

Obesogenic environment. Except obesogens, there are some other obesogenic environments, for example, prolonged light use and air pollution.

Prolonged light use is another reason for obesity, which is easy to neglect. Humans sense change of light and keep circadian rhythm by the 5-HT and melatonin from the pineal body, which lies between the diencephalon and the thalamus. Artificial lighting increases by 6 percent annual worldwide, and this disrupts normal circadian rhythm, causing adverse events such as insomnia, anxiety and physiological changes [13]. Increasing exposure to artificial light increases body adiposity via impaired activity of brown adipose tissue (BAT). Compared with regular 12 hours light, 16 and 24-hours of artificial light exposure increased adipose tissue, at the same level of locomotor activity and food uptake [14]. Researchers from Leiden University Medical Center proposed that BAT activity plays an important role in the association between adiposity and disturbed circadian rhythm, and activation of BAT is likely to overcome the adverse metabolic consequences of altered circadian rhythmicity.

Air pollution could decrease vitamin D in the body and cause obesity indirectly. WHO announced that nearly $80 \%$ of the population worldwide are faced with air pollution exceeding the limit . Air pollution would absorb UVB radiation, hence reducing sunlight reaching creatures on earth. Considering that humans need UVB to synthesize vitamin D, air pollution would contribute to vitamin $\mathrm{D}$ deficiency. Adipose tissue express vitamin $\mathrm{D}$ receptors widely, through which vitamin $\mathrm{D}$ would inhibit the differentiation of pre-adipocytes [15].

Viral infections could contribute to obesity.In various experimental models, several pathogenes could contribute to obesity --called infectobesity. These agents alter metabolic function in cells and are associated with human obesity [16].

\section{Preventions}

No longer can we blame obesity on personal choices or lifestyle changes only, but we have to consider that environmental chemicals are also contributing to this mounting human health problem. Against similar genetic backgrounds, same energy uptake and lifestyle, it would be useful to reduce or delay the process of overweight or obesity to a large extent by taking some action to avoid these hazards.

Firstly, government and policy should reduce obesogens use in food and output from chemical products from the source.

Secondly, individuals need to reduce exposure to obesogens and other obesogenic environments.Products labeled as ' $\mathrm{x}$-free'( $\mathrm{x}$ could refer to any obesogens) should be your first choice. Using less personal care products would be of benefit to adolescent girl. Eating fresh food instead of processed food would decrease exposure to obesogens and then reduce risks of being obese. Balance the types and proportions of intestinal flora. Taking prebiotics: 'The selective stimulation of growth and / or activity(ies) of one or a limited number of microbial genus(era) / species in the gut microbiota 
that confer(s) health benefits to the host 'may also limit the form of obesity. There has been significant research oninulin-type fructans (ITF). ITFs improve hepatic insulin sensitivity and increase plasma insulin in diet-induced diabetes and obesity [17]. In order to keep from these detrimental environment factors, one should also reduce light use.

Thirdly, it is urgent to carry out mass education. All actions would not make any process unless the public have a basic knowledge about obesogens and obesogenic environment and their negative influence.

\section{Summary}

Knowledge about obesogen is still in its infancy since it was raised no more than two decades years ago. Because the available evidence is based on cell-based experiments and animal experiments, the exact mechanisms in obesogen-induced changes in humans are yet to be further experimentally demonstrated. It may be possible to identify more obesogens by high-throughput screening approaches, and carry out human epidemiology studies to deepen the understanding of these chemicals called obesogens.

\section{Acknowledgements}

The authors are grateful to Rongzhu Lu for instruction and English editing. And thanks for the support from below funds:

1. 2016 China Ministry of Education awarded the second batch of "Top Brand Curricula" of English teaching program-Pharmacology

2. 2015 Jiangsu Province awarded the excellent curricula of Englishi teaching program for overseas students-Pharmacology

3. The key project of education reform program in Jiangsu University in 2015, item No. 2015JGZD027

4. 2012 Jiangsu Province Postdoc Fund 1202041C

\section{References}

[1] Grün F1, Blumberg B.Environmental obesogens: organotins and endocrine disruption via nuclear receptor signaling.Endocrinology. 2006 Jun;147(6 Suppl): S50-5.

[2] Masuno H, Iwanami J, Kidani T, Sakayama K, Honda K. Bisphenol a accelerates terminal differentiation of 3T3-L1 cells into adipocytes through the phosphatidylinositol 3-kinase pathway. Toxicol Sci. 2005 Apr;84(2):319-27

[3] Iguchi T, Watanabe H, Ohta Y, Blumberg B. Developmental effects: oestrogen-induced vaginal changes and organotin-induced adipogenesis. Int J Androl. 2008 Apr;31(2):263-8.

[4] Hao CJ, Cheng XJ, Xia HF, Ma X. The endocrine disruptor 4-nonylphenol promotes adipocyte differentiation and induces obesity in mice. Cell Physiol Biochem. 2012;30(2):382-94.

[5] Desvergne B, Feige JN, Casals-Casas C. PPAR-mediated activity of phthalates: A link to the obesity epidemic? Mol Cell Endocrinol. 2009 May 25;304(1-2):43-8.

[6] Matsushita M, Egashira N, Harada S, Okuno R, Mishima K, Iwasaki K, Nishimura R, Fujiwara M. Perospirone, a novel antipsychotic drug, inhibits marble-burying behavior via 5-HT1A receptor in mice: implications for obsessive-compulsive disorder. J Pharmacol Sci. 2005 Oct;99(2):154-9.

[7] Yan Z, Zhang H1, Maher C, Arteaga-Solis E, Champagne FA, Wu L, McDonald JD, Yan B, Schwartz GJ, Miller RL. Prenatal polycyclic aromatic hydrocarbon, adiposity, peroxisome proliferator-activated receptor (PPAR) $\gamma$ methylation in offspring, grand-offspring mice. PLoS One. 2014 Oct 27;9(10): e110706.

[8] Neel BA, Brady MJ, Sargis RM. The endocrine disrupting chemical tolylfluanid alters adipocyte metabolism via glucocorticoid receptor activation. Mol Endocrinol. 2013 Mar;27(3):394-406. 
[9] Newbold RR, Padilla-Banks E, Jefferson WN, Heindel JJ. Effects of endocrine disruptors on obesity. Int J Androl. 2008 Apr;31(2):201-8.

[10] Harley KG, Berger K, Rauch S, Kogut K, Claus Henn B, Calafat AM, Huen K, Eskenazi B, Holland N. Association of prenatal urinary phthalate metabolite concentrations and childhood BMI and obesity. Pediatr Res. 2017 Sep;82(3):405-415.

[11] Yamamoto A, Wada O, Ono T, Ono H, Manabe S, Ishikawa S. Cadmium-induced stimulation of lipogenesis from glucose in rat adipocytes. Biochem J. 1984 May 1;219(3):979-84.

[12] Buck J, Levin LR. Physiological sensing of carbon dioxide/bicarbonate/pH via cyclic nucleotide signaling. Sensors (Basel). 2011;11(2):2112-28.

[13] Harb F, Hidalgo MP, Martau B. Lack of exposure to natural light in the workspace is associated with physiological, sleep and depressive symptoms. Chronobiol Int. 2015 Apr;32(3):368-75.

[14] Kooijman S, van den Berg R, Ramkisoensing A, Boon MR, Kuipers EN, Loef M, Zonneveld TC, Lucassen EA, Sips HC, Chatzispyrou IA, Houtkooper RH, Meijer JH, Coomans CP, Biermasz NR, Rensen PC. Prolonged daily light exposure increases body fat mass through attenuation of brown adipose tissue activity. Proc Natl Acad Sci U S A. 2015 May 26;112(21):6748-53.

[15] Kurylowicz A. Impact of Air Pollution on Vitamin D Status and Related Health Conse-quences[M]// The Impact of Air Pollution on Health, Economy, Environment and Agricultural Sources. InTech, 2011.

[16] Breton J, Massart S, Vandamme P, De Brandt E, Pot B, Foligné B. Ecotoxicology inside the gut: impact of heavy metals on the mouse microbiome. BMC Pharmacol Toxicol. 2013 Dec 11;14:62.

[17] Roberfroid M, Gibson GR, Hoyles L, McCartney AL, Rastall R, Rowland I, Wolvers D, Watzl B, Szajewska H, Stahl B, Guarner F, Respondek F, Whelan K, Coxam V, Davicco MJ, Léotoing L, Wittrant Y, Delzenne NM, Cani PD, Neyrinck AM, Meheust A. Prebiotic effects: metabolic and health benefits. Br J Nutr. 2010 Aug;104 Suppl 2:S1-63. 